\title{
Low-k Dielectric- A Potential Solution for Crosstalk Induced Signal Integrity issues in SWCNT Interconnects
}

\author{
Nisha Kuruvilla ${ }^{1}$, Amrutha S.R. ${ }^{2}$ and J.P. Raina ${ }^{3}$ \\ ${ }^{1 \& 3}$ (Center of Nanotechnology, VIT University, Vellor, Tamilnadu, India) \\ ${ }^{1 \& 2}$ (Electronics and Communication Department, College of Engineering, Chengannur, Kerala, India)
}

\begin{abstract}
Crosstalk creates signal integrity issues due to capacitive coupling between adjacent interconnect wires and it is a matter of concern in high frequency interconnects. The reported work on crosstalk induced signal integrity issues in CNT interconnects till date were assuming the value of coupling capacitance as equivalent to the coupling effect between metal interconnects of same dimensions. This work tries to fill that gap; by analyzing crosstalk in bundled SWCNTs with a better model for extracting inter bundle real life coupling capacitances. This work also proposes a novel idea of reducing crosstalk effects by using low-k dielectric materials as isolation between adjacent nets. It is found that compact bundles separated by low-k dielectric can reduce crosstalk effect considerably.
\end{abstract}

Keywords - Crosstalk, signal integrity, SWCNT, MWCNT, Mixed CNT

\section{INTRODUCTION}

CNTs are proposed as an alternative technology for the future interconnects in integrated circuits against copper technology, which was the dominant interconnect technology from 1990 [1]. As these interconnects intended to carry signal frequency from upper Giga Hertz to Tera-Hertz, signal integrity issues due to crosstalk will be a primary concern. The work on crosstalk analysis in multiple SWCNTs was initiated by [2]. All the works reported till date on this issue [ 2-3, 5-6], assumed that the value of coupling capacitance as equivalent to the coupling effect between metal interconnects of same dimensions or never taken the inter bundle coupling capacitance into account as in [4]. As material properties of copper are widely varying from CNTs, a need for real life estimation is inevitable. This work aims to fill that gap, by calculating the inter bundle capacitance value with the help of validated empirical relationships for CNT interconnects [1].

In real life the magnitude of crosstalk induced effect is found to be increasing than the earlier reported values [26]. This work proposes a remedial solution for crosstalk induced signal integrity issues by using low-k materials for isolation between adjacent nets. This work also quantifies the reduction of crosstalk effect on using low-k dielectrics as an isolation material.

\section{Crosstalk Coupling CaPaCitance EFFECT Modeling AND ITS ANALYSIS METHODOLOGY}

The magnitude of real life value of coupling capacitive $\left(\mathrm{C}_{\mathrm{C}}\right)$ effect existing between adjacent $\mathrm{CNT}$ bundles can be estimated using the model proposed in [1]. This model can be mapped onto this application as it is capable of providing capacitance between such geometries. In order to analyze the influencing factors which affects the crosstalk coupled voltage on adjacent wires, a geometry of three parallel SWCNT bundle interconnects as shown in Fig. 1 has been used.

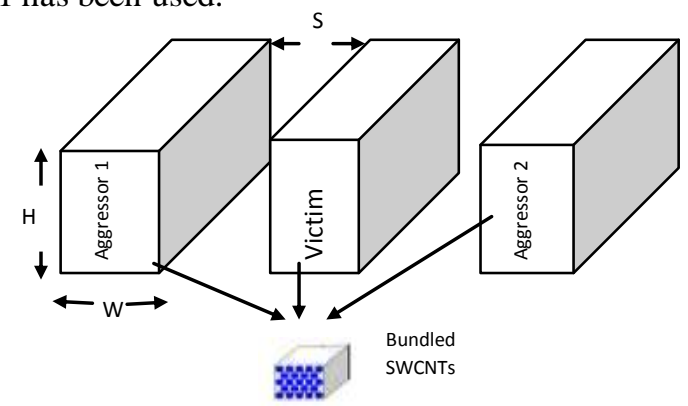

Fig.1 Three parallel SWCNT bundle interconnects geometry for the signal integrity analysis

The basic expression for estimating electrostatic capacitance between interconnects made of single SWCNT and the ground plane is as in equation 1 ,

$C_{E} \cong \frac{2 \pi \varepsilon}{\ln \left(\frac{y}{d}\right)}$ 
where ' $d$ ' is the diameter of the CNT and ' $y$ ' is the distance between CNT and the ground plane.

The model for extracting the coupling capacitance between adjacent bundles of CNTs can be summarized as in equation 2, [1]

$C_{E}^{\text {bundle }}=2 C_{E n}+\frac{n_{W}-2}{2} C_{E f}+\frac{3\left(n_{H}-2\right)}{5} C_{E n}$

$\mathrm{n}_{\mathrm{H}}$ is the number of "rows" in the interconnect bundle, $\mathrm{n}_{\mathrm{W}}$ is the number of "columns". $C_{E n}$ and $C_{E f}$ are the near and far neighbour inter bundle capacitive effects. In case of $C_{E n}$ the ' $y$ ' in equation (1) will be equivalent to spacing between adjacent bundles. While in the case of $C_{E f}$, it will be equivalent to ( $\mathrm{s}+\mathrm{w}$ ) where w is the width of the bundle[1].This is a modelled expression which was validated by K Banerjee's group with result obtained from FastCap [1]

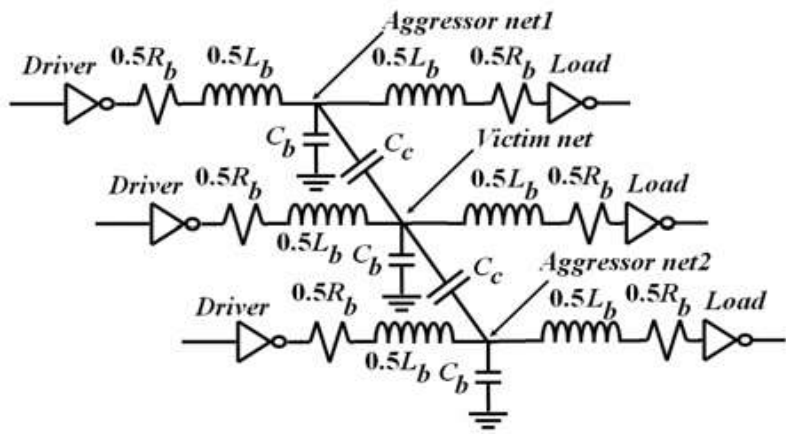

Fig. 2 Symmetrical T-structured ESC model for crosstalk analysis [9]

The entire crosstalk analysis was done with the help of symmetrical T-structured ESC model as shown in Fig. 2. The RLC parameter values were calculated as per [1]

\section{Crosstalk Effect In Bundled SWCNTs Under Real Life Scenario With High-K Dielectric Isolation}

Crosstalk induced signal integrity issues were analyzed by considering silicon dioxide as the inter bundle dielectric isolation material. In this section a comparative analysis of crosstalk induced effects between bundles of SWCNTs with coupling capacitance (Cc) equivalent to that of copper as in work [2-6] against Cc calculated from equation 2,( i.e. CNT coupling under real life condition). A measure of rise time, fall time, overshoot and undershoot voltages due to crosstalk were evaluated. The worst case variations in rise time were calculated, by comparing between the rise time variations of victim wire due to aggressor switching to that of grounded aggressor conditions. Similarly fall time variations were calculated by the same method. For overshoot and undershoot analysis, the victim net is kept at fixed logic 1 and 0 levels, respectively while the aggressor nets are switched from logic $1 \rightarrow 0$ and logic $0 \rightarrow 1$ respectively. A consolidate comparison of parameters are given in Table 1. On comparing crosstalk induced signal integrity issues with the published work [2-6], signal integrity issues in CNTs are found to be a mounting problem than reported in the literatures. The magnitudes of variations in rise time, fall time, overshoot and undershoot voltages are found to be increasing. Hence a novel solution is required for the reduction of crosstalk in bundled SWCNT interconnects. This can be achieved by using low-k dielectric as isolation between bundles.

Table 1: Comparison of real life signal integrity issues in CNT interconnects due to crosstalk

\begin{tabular}{|c|c|c|c|c|}
\hline \multirow[b]{2}{*}{ Parameter } & \multicolumn{2}{|c|}{ Length $=1 \mu \mathrm{m}$} & \multicolumn{2}{|c|}{ Length $=10 \mu \mathrm{m}$} \\
\hline & $\begin{array}{l}\text { Signal } \\
\text { integrity } \\
\text { issues with } \\
\mathrm{C}_{\mathrm{c}} \quad(\mathrm{Cu}) \\
{[5]}\end{array}$ & $\begin{array}{l}\text { Signal } \\
\text { integrity } \\
\text { issues } \\
\text { with } \mathrm{C}_{\mathrm{c}} \\
(\mathrm{CNT})\end{array}$ & $\begin{array}{l}\text { Signal } \\
\text { integrity } \\
\text { issues with } \\
\mathrm{C}_{\mathrm{c}}(\mathrm{Cu}) \text { [5] }\end{array}$ & $\begin{array}{l}\text { Signal } \\
\text { integrity } \\
\text { issues with } \\
\mathrm{C}_{\mathrm{c}}(\mathrm{CNT})\end{array}$ \\
\hline Rise Time reduction (ps) & 0.011 & 1.249 & 0.3805 & 20.301 \\
\hline Fall Time reduction (ps) & 0.011 & 1.242 & 0.3696 & 20.083 \\
\hline Overshoot (V) & 0.0132 & 0.3467 & 0.0372 & 0.4385 \\
\hline Undershoot $(\mathrm{V})$ & 0.0133 & 0.3467 & 0.0372 & 0.4385 \\
\hline
\end{tabular}


Low-k Dielectric- A Potential Solution for Crosstalk Induced Signal Integrity issues in SWCNT IV. LOW-K DIELECTRIC- A POTENTIAL SOLUTION FOR THE REDUCTION OF CROSSTALK

It is possible to reduce coupling capacitance if the relative dielectric constant $(\mathrm{k})$ of the insulating material is reduced. This work hence attempted to quantify variation in crosstalk effect as a function of various dielectric filling between the adjacent interconnects. The different dielectric materials considered for the estimation of crosstalk effect includes, Silicon dioxide $(\mathrm{SiO} 2)\left(\varepsilon_{\mathrm{r}}=3.9\right)$, polybenzoxazole $(\mathrm{PBO})\left(\varepsilon_{\mathrm{r}}=2.7\right)$, silicon dioxide mixed with fluorine, hydrogen or alkyl group $(\mathrm{CH})\left(\varepsilon_{\mathrm{r}}=2.5\right)$ and porous silicon $\operatorname{dioxide}\left(\varepsilon_{\mathrm{r}}=2\right)$. The various values of $\mathrm{Cc}$ under different dielectric filling conditions were calculated using Equation 2. These values are used for the estimation of transient analysis of densely/ sparsely packed SWCNT for length $1 \mu \mathrm{m}$ and $10 \mu \mathrm{m}$. Fig. 3 shows that crosstalk induced signal integrity issues are found to be reducing by $15 \%$ in the case of dense bundles when low-k dielectric filling was used instead of $\mathrm{SiO} 2$ for interconnect of $1 \mu \mathrm{m}$ length.

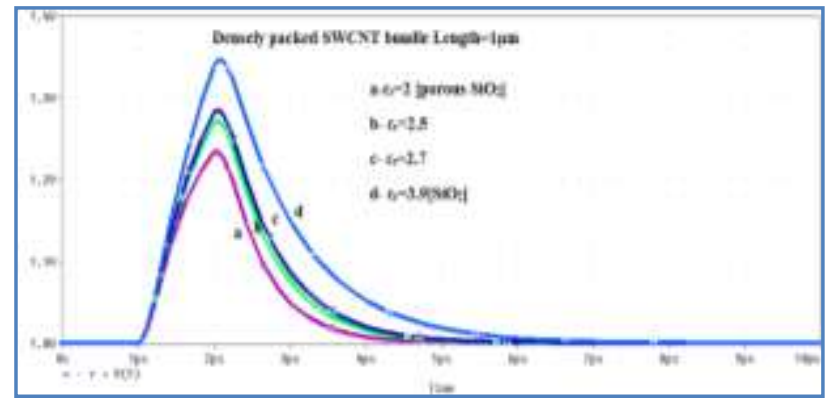

Fig. 3 Transient analysis of crosstalk induced noise in densely packed SWCNT local interconnects

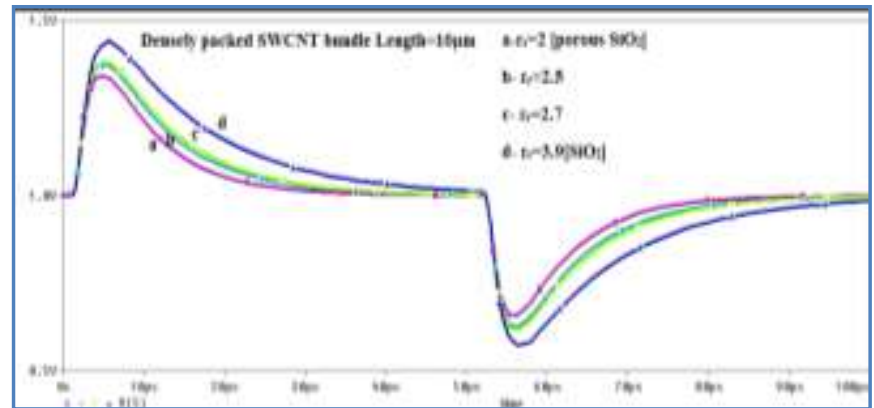

Fig. 4 Transient analysis of crosstalk induced noise in densely packed SWCNT global interconnects

Similar conditions were observed in global interconnects also. In case of global interconnects of length $10 \mu \mathrm{m}$ or more, crosstalk induced signals were unable to settle down within the permitted time period, for the high-k dielectric filling as shown in Fig. 4. Hence usage of low-k dielectric filling is inevitable along with bundled CNT interconnects for its reliable performance.

\section{CONCLUSION}

Crosstalk induced signal integrity issues in bundled CNT interconnect is found to be larger than the reported values available in the exiting literature. This was due to the wrong assumption of coupling capacitance value. As CNTs are only one available material option for Terahertz interconnects, the reliable operation from the same can be obtained only when inter dielectric filling is of low-k material. Such CNT interconnect structures will be an optimum solution for the reduction of crosstalk induced signal integrity issues in SWCNT interconnects.

[1] Banerjee, K. and Srivastava, N., Performance Analysis of Carbon Nanotube interconnects for VLSI Applications, IEEE Int. Computer Aided Design Conf., San Jose, CA, 2005, 383-390

[2] Rossi, D. , Cazeaux, J. M. , Metra, C. and Lombardi, Modeling crosstalk effects in CNT bus architectures, IEEE Trans. Nanotechnology, 6(2), 2005, 133-145

[3] Shao-Ning Pu, Wen-Yan Yin, Jun-Fa Mao, and Qing H. Liu, Crosstalk Prediction of Single- and Double-Walled CarbonNanotube (SWCNT/DWCNT) Bundle Interconnects, IEEE Trans. Electron Devices, 56 (4), 2009, 560-568

[4] Marcello D'Amore, Maria Sabrina Sarto, and Alessio Tamburrano, Fast Transient Analysis of Next-Generation Interconnects Based on Carbon Nanotubes, IEEE Trans. Electromagn. Compat.,52(2), 2010, 496- 503

[5] Debaprasad Das, Hafzur Rahaman, Analysis of crosstalk in single and multiwall carbon Nanotube interconnects and its impact on gate oxide reliability', IEEE Trans. Nanotechnology,10(6), 2011, 1362-1370

[6] Kailiang Zhang, Bo Tian, Xiaosong Zhu, Fang Wang and Jun Wei, Crosstalk analysis of carbon nanotube bundl Interconnects', Nanoscale Research Letters , 2012, 7:138. 